\title{
Incidence of Medication-Treated Depression and Anxiety Associated with Long-Term Cancer, Cardiovascular Disease, Diabetes and Osteoarthritis in Community-dwelling Women and Men
}

\author{
Xianwen Shang, ${ }^{\mathrm{a}, \mathrm{c}, \mathrm{d}}$, Wei Peng ${ }^{\mathrm{e}}$, Edward Hill ${ }^{\mathrm{c,d}}$, Cassandra Szoeke ${ }^{\mathrm{c}, \mathrm{d}}$, Mingguang He $\mathrm{He}^{\mathrm{a}, \mathrm{f}, \mathrm{g}, * *}$, \\ Lei Zhanga,b,h,i,*,1 \\ ${ }^{a}$ Centre for Eye Research Australia, Royal Victorian Eye and Ear Hospital, Melbourne, Australia \\ ${ }^{\mathrm{b}}$ China-Australia Joint Research Center for Infectious Diseases, School of Public Health, Xi'an Jiaotong University Health Science Centre, Xi'an, Shaanxi, China \\ ' School of Behavioural and Health Sciences, Australian Catholic University, Australia \\ ${ }^{\mathrm{d}}$ Department of Medicine (Royal Melbourne Hospital), The University of Melbourne, Australia \\ e Research Centre for Data Analytics and Cognition, La Trobe University, Melbourne, Australia \\ ${ }^{\mathrm{f}}$ Ophthalmology, Department of Surgery, University of Melbourne, Melbourne, Australia \\ State Key Laboratory of Ophthalmology, Zhongshan Ophthalmic Centre, Sun Yat-sen University, Guangzhou, China \\ ${ }^{\mathrm{h}}$ Melbourne Sexual Health Centre, Alfred Health, Melbourne, Australia \\ i Department of Epidemiology and Biostatistics, College of Public Health, Zhengzhou University, Zhengzhou 450001, Henan, China
}

\section{A R T I C L E I N F O}

\section{Article history:}

Received 23 May 2019

Revised 16 July 2019

Accepted 19 August 2019

Available online 30 August 2019

\section{Keywords:}

Cardiovascular disease

Cancer

Diabetes

Osteoarthritis

Depression

Anxiety

\begin{abstract}
A B S T R A C T
Background: Long-term cancer, cardiovascular disease, diabetes and osteoarthritis may increase the risk of mental disorders, but which was more harmful and whether the associations differed between genders is unclear.

Methods: We included 115,094 participants (54.3\% women) aged 45-64 years from the 45 and Up Study who were free of depression, anxiety, and Parkinson's disease at baseline (2006-2009). The incidence of depression and anxiety was identified using claim databases during follow-up until December 2016. Cox regression models were used to examine the association of cancer, cardiovascular disease, diabetes, and osteoarthritis at baseline with incident depression and anxiety.

Findings: During a mean eight-year follow-up (958,785 person-year), the cumulative incidence of depression and anxiety was $12.5 \%$ and $5.9 \%$ in the healthy population. Hazard ratios ([HRs] (95\% CI) versus healthy population) for incident depression associated with long-term cancer, cardiovascular disease, diabetes, and osteoarthritis were 1.19 (95\% CI: 1.13-1.25), 1.08 (1.00-1.16)), 1.18 (1.09-1.28), and 1.94 (1.802.10), respectively. The corresponding HRs (95\% CIs) for incident anxiety were 1.11 (1.03-1.20), 1.26 (1.141.39), 1.10 (0.98-1.24), and $2.01(1.80-2.23)$, respectively. The positive association between cancer and incident depression was more evident in men (HR (95\% CI): 1.24 (1.13-1.35) than in women (1.14 (1.071.21). Long-term diabetes was an independent risk factor for incident anxiety in men (1.21 (1.02-1.44) but not in women $(1.09(0.93-1.28))$.

Interpretation: Long-term osteoarthritis, cardiovascular disease, and cancer were independent risk factors for incident depression and anxiety in both genders with osteoarthritis having the highest relative risk.
\end{abstract}

(c) 2019 Published by Elsevier Ltd. This is an open access article under the CC BY-NC-ND license.

(http://creativecommons.org/licenses/by-nc-nd/4.0/)
* Correspondence to: L. Zhang, China-Australia Joint Research Center for Infectious Diseases, School of Public Health, Xi'an Jiaotong University Health Science Centre, Xi'an, Shaanxi, China.

** Correspondence to: M. He, Centre for Eye Research Australia, University of Melbourne, Level 7, 32 Gisborne Street, East Melbourne, VIC 3002, Australia.

E-mail addresses: mingguang.he@unimelb.edu.au (M. He), Lei.zhang1@xjtu.edu.cn (L. Zhang).

1 First Corresponding author.

\section{Introduction}

Cardiovascular disease (CVD) and cancer were the two leading causes of global mortality in 2015, which accounted for 17.9 million and 8.7 million deaths, respectively [1]. Death from diabetes rose from ninth in 2006 to seventh in 2016 for men and from eighth in 2006 to seventh in 2016 for women in the rank of the leading cause of death in Australia [2]. Although osteoarthritis is not a 


\section{Research in context}

\section{Evidence before this study}

A literature search was conducted at PubMed (up to January 21, 2019) using the search terms "cancer", "cardiovascular disease", "diabetes", or "osteoarthritis" combined with "depression", "anxiety", or "mental disorders" with no language restriction. Although cancer, cardiovascular disease, diabetes, and osteoarthritis have been individually linked to depression or anxiety, it is unclear which condition is more harmful for mental health or whether the association differed by genders.

\section{Added values of this study}

To our knowledge, this is the first study to comprehensively examine the association of long-term cancer, cardiovascular disease, diabetes and osteoarthritis with incident depression and anxiety at different time points of followup in a community-dwelling population with a large sample size and long-term follow-up. This study also analysed the association between physical conditions and incident depression and anxiety in women compared with men. During follow-up (958,785 person-year), the cumulative incidence of depression was $12.5 \%$ in healthy population and 15.1\% (Hazard ratio [HR] $(95 \% \mathrm{CI})$ VS healthy population: 1.19 (1.13-1.25)), 15.1\% (1.08 (1.00-1.16)), 16.7\% (1.18 (1.09$1.28)$ ), and $25.4 \%(1.94(1.80-2.10))$ in individuals with cancer, cardiovascular disease, diabetes, and osteoarthritis, respectively. The corresponding incidence of anxiety was 5.9\%, 6.8\% (1.11 (1.03-1.20)), 8.7\% (1.26 (1.14-1.39)), 7.6\% (1.10 (0.98$1.24)$ ), and $13.4 \%(2.01(1.80-2.23))$, respectively. Osteoarthritis had the highest risk for both incident depression and anxiety among four conditions. The association of cancer with incident depression and diabetes with incident anxiety was more evident in men than women. Other associations did not differ between men and women. Our results were consistent for different time points of follow-up.

\section{Implications of the all the available evidence}

Identifying the association between long-term physical conditions and incident depression and anxiety may help target individuals who were at most risk of mental disorders. Our findings highlight the importance of depression and anxiety screen in individuals with those long-term physical conditions. Anxiety screen deserves more concern in men with long-term diabetes.

leading cause of death, its high prevalence and profound impact on disability have posed a tremendous burden on the healthcare system [3,4]. These major chronic conditions have been observed to be commonly clustered with mental disorders [2], which resulted in an excess risk of deaths from cancer [5], CVD [6], and diabetes [7], and disability caused by arthritis [8].

Mental disorders have been demonstrated to be risk factors for cancer, CVD, diabetes, and osteoarthritis [9-14], yet whether these physical conditions are associated with increased risks of mental disorders is less known. Recently, an increasing number of studies used claim data to analyse the risk of mental disorders associated with long-term physical conditions $[15,16]$. However, these studies were mostly restricted to focusing on one of these physical conditions and it is unclear which condition is comparatively more harmful for mental health. Most previous research studies targeted population in a wide range of age [17], only a few investigated middle-age adults, which is an important period for early prevention of mental disorders. Furthermore, women are more likely to be affected by a common mental disorder at some time during their life than men [18]. The gender gap in the prevalence of men- tal disorders may be partly attributed to the fact that women are more likely to develop mental disorders following chronic conditions compared with men. Whether the positive association between mental disorders and physical conditions was more evident in women remains to be explored.

We prospectively examined the incidence of depression and anxiety associated with long-term cancer, CVD, diabetes, and osteoarthritis. We then analysed whether these associations were more likely to be manifested in women than men.

\section{Methods}

\subsection{Participants}

The 45 and Up Study is a prospective study of 266,896 participants aged 45 years and over from New South Wales (NSW) [19]. Participants were randomly sampled from the general population through the Department of Human Services (formerly Medicare Australia) enrolment database, and an 18\% response rate was achieved, corresponding to $11 \%$ of the entire NSW population in this age group [20]. Baseline data including lifestyle behaviours, medical history, family history of chronic conditions, socioeconomic status, and geographic factors were collected between 2006 and 2009. The 45 and Up study has ethical approval from the UNSW Human Research Ethics Committee. The study protocol was approved by the Royal Victorian Eye and Ear Hospital Human Research Ethics Committee. Participants provided consent to followup and link their data to routine health datasets.

This analysis excluded participants with depression, anxiety, dementia, Parkinson's disease, and concurrence of two or more of cancer, CVD, diabetes, and osteoarthritis based on self-reported history of previous diagnosis, Medicare Benefits Schedule (MBS) or Pharmaceutical Benefits Scheme (PBS) claims. We also excluded participants with Department of Veterans' Affairs cards because information on these people is not included in PBS or MBS, those aged 65 years or older, or those who needed help with daily tasks because of long-term illness disability at baseline (Fig. S1).

\subsection{Independent Variables}

Medical history of heart disease, stroke, diabetes, cancer, osteoarthritis, hypertension, Parkinson's disease, depression, asthma, and hip fracture was self-reported. Individuals were divided into five groups: cancer (those who reported a diagnosis of cancer or those with procedures or medications before or until baseline recorded by MBS or PBS claims), CVD (self-reported history of previous diagnosis, or MBS claims), diabetes (self-reported history of previous diagnosis or PBS claims), osteoarthritis (self-reported history of previous diagnosis or PBS claims), and healthy population without any of the four conditions.

\subsection{Covariate Variables}

Demographic information including age, gender, ethnicity, income, and education was self-reported. Body mass index was calculated based on self-reported height and weight. Psychological distress was accessed using the Kessler-10 scale [21], and scores ranged from 10 to 50, with the following categories: low [10,11], mild [12-15], moderate [16-21] and high (22-50) psychological distress. Family history of heart disease, stroke, hypertension, diabetes, cancer, Alzheimer's disease, Parkinson's disease, depression, and arthritis was self-reported.

\subsection{Outcome Variables}

Baseline data were linked to MBS and PBS data (July 01, 2004December 31, 2016) by the Sax Institute using a unique identi- 
fier provided by the Department of Human Services. The incidence of depression (prescription for antidepressants) and anxiety (anxiolytics) during follow-up was determined by medications claimed by study participants via PBS (codes are in Table S1). Person-year for participants was calculated from the date of data collected at baseline to the date of depression/anxiety onset, or the end of the follow-up on December 31, 2016, whichever came first.

\subsection{Statistical Analysis}

Descriptive data were summarised as frequency and percentage, and Chi-square test was used to evaluate the difference of baseline characteristics across chronic condition groups.

The associations of long-term cancer, CVD, diabetes, and osteoarthritis with the incidence of depression and anxiety were assessed using Cox regression models. The multivariable-analysis was adjusted for age, gender, the country of birth, income, education, family history of conditions, hypertension, dyslipidaemia, asthma, BMI, and psychological distress. We also used Cox proportional regression models to assess the interaction between gender and physical conditions for incident depression and anxiety. BenjaminHochberg procedure was used to control the false discovery rate at level $5 \%$ for multiple comparisons [22].

Sensitivity analysis was conducted to examine whether women had a higher incidence of mental disorders (depression and/or anxiety) than men in the healthy population and those with cancer, CVD, diabetes, or osteoarthritis. Furthermore, we analysed whether each of the four conditions was associated with increased risks of developing mental disorders in men and women. Third, we examined whether major types of cancer including breast cancer, prostate cancer, melanoma, and other cancer were predictive of incident depression, anxiety, and mental disorders. Fourth, we analysed whether multimorbidity (concurrence of two or more of cancer, CVD, diabetes, and osteoarthritis) was associated with increased risk of depression, anxiety, and mental disorders.

All analyses with the use of two-tailed tests were performed using SAS version 9.4 (SAS Institute Inc.).

\section{Results}

\subsection{Participant Characteristics}

We included 115,094 participants (54.3\% women) with a mean follow-up of $8.3 \pm 1.8$ (range $7.0-11.5$ ) years in the final analysis, among which $11,057,5348,4348$, and 5415 were individuals with cancer, CVD, diabetes, and osteoarthritis and 88,926 were healthy participants (Table 1). Women had a higher prevalence of cancer (10.6\% in women, $8.5 \%$ in men) and osteoarthritis $(5.6 \%, 3.7 \%)$ but a lower prevalence of CVD $(3.0 \%, 6.6 \%)$ and diabetes $(3.0 \%, 4.7 \%)$ than men at baseline (all P-value $<0.0001$ ). Healthy individuals were more likely to be younger, have higher income and education, and have lower baseline prevalence of hypertension, dyslipidaemia, and overweight/obesity compared to those with a chronic condition (all P-value $<0.0001$ ).

\subsection{Incidence of Depression and Anxiety}

During follow-up (958,785 person-year), the cumulative incidence of depression was $12.5 \%$ in healthy population and $15.1 \%$ (Hazard ratio [HR] $(95 \% \mathrm{CI})$ VS healthy population: 1.19 (1.13$1.25)$ ), $15.1 \%$ (1.08 (1.00-1.16)), $16.7 \%$ (1.18 (1.09-1.28)), and $25.4 \%$ (1.94 (1.80-2.10)) in individuals with cancer, CVD, diabetes, or osteoarthritis, respectively. The corresponding incidence of anxiety was $5.9 \%, 6.8 \%(1.11(1.03-1.20)), 8.7 \%$ (1.26 (1.14-1.39)), 7.6\% (1.10 (0.98-1.24)), and 13.4\% (2.01 (1.80-2.23)), respectively (Fig. 1).

\subsection{Incidence of Depression and Anxiety by Gender}

Women had a consistently higher incidence of depression than men in healthy population (14.8\% in women, $9.8 \%$ in men, HR $(95 \%$ $\mathrm{CI}): 1.47(1.42-1.53))$, and those with cancer $(17.0 \%, 12.4 \%, 1.39$ (1.25-1.55)) CVD (19.5\%, 12.7\%, 1.55 (1.34-1.79)), diabetes (20.6\%, $13.7 \%, 1.43(1.23-1.66))$, or osteoarthritis $(28.4 \%, 20.1 \%, 1.53$ (1.351.72)). The corresponding HRs (95\% CIs) for anxiety were 1.56 (1.47-1.66), 1.48 (1.26-1.74), 1.68 (1.38-2.03), 1.29 (1.03-1.61), and 1.39 (1.18-1.64), respectively (Fig. 2). This association was significant across different time points (Fig. S2).

\subsection{Incidence of Depression and Anxiety Associated With Long-term Cancer, CVD, Diabetes, and Osteoarthritis in Women Compared With Men}

In the multivariable-analysis, the positive association between any of cancer, CVD, diabetes, and osteoarthritis and incident depression was significant in both men and women. This association was significant across different time points although the HRs for depression decreased with increased follow-up period. The HRs (CIs) for incident depression associated with baseline cancer in men were 1.55 (1.31-1.84), 1.51 (1.33-1.72), 1.32 (1.19-1.46) at year 3,5 , and 7, respectively, whereas the corresponding HRs (CIs) for women were 1.20 (1.06-1.36), 1.18 (1.07-1.29), and 1.17 (1.08-1.25), respectively. This association was more evident in men at year 3 , 5 , and 7 of follow-up than women and did not differ between genders at year 9 and 11 of follow-up. The magnitude of the association between CVD, diabetes and osteoarthritis and incident depression was not different between genders (Fig. 3).

The associations of baseline CVD and osteoarthritis with incident anxiety were significant in both men and women across different time points, and the magnitude of these associations did not differ between genders. Baseline cancer was an independent risk factor for incident anxiety in men from year 3 to 11 of followup whereas the association between baseline cancer and incident anxiety in women was significant from year 3 to 7 of follow-up but not significant at the end of the follow-up. Baseline diabetes was an independent risk factor for incident anxiety in men across different time points but was not independently associated with incident anxiety at any time point in women (Fig. 4).

\subsection{Sensitivity Analysis}

Women had a higher incidence of mental disorders than men in the healthy population, and individuals with cancer, CVD, diabetes, or osteoarthritis (Fig. S3). Each of the four chronic conditions was associated with an increased risk of mental disorders in both men and women across different time points (Fig. S4). Baseline melanoma was associated with an increased risk of incident depression in men only. Baseline breast cancer was an independent risk factor for incident depression in women so was baseline prostate cancer in men. Other cancer at baseline was associated with a higher incidence of depression in both men and women whereas the magnitude was greater in men (Fig. S5). Baseline prostate cancer was associated with a higher incidence of anxiety in men (Fig. S6). Other cancer at baseline was associated with a higher incidence of mental disorders in both men and women (Fig. S7). Individuals with multimorbidity at baseline had higher incidence of depression, anxiety, and mental disorders and this association did not differ between women and men (Fig. S8).

\section{Discussion}

We found women had a higher incidence of depression and anxiety than men and this association was significant in the 
Table 1

Baseline characteristics of study participants by chronic conditions.

\begin{tabular}{|c|c|c|c|c|c|c|}
\hline & \multicolumn{5}{|l|}{ Chronic conditions } & \multirow[b]{2}{*}{ P value } \\
\hline & Healthy participants ${ }^{a}$ & Cancer & CVD & Diabetes & Osteoarthritis & \\
\hline Gender & & & & & & 0.0004 \\
\hline Men & $40,338(45.4 \%)$ & $4453(40.3 \%)$ & $3454(64.6 \%)$ & $2467(56.7 \%)$ & $1922(35.5 \%)$ & \\
\hline Women & $48,588(54.6 \%)$ & $6604(59.7 \%)$ & $1894(35.4 \%)$ & $1881(43.3 \%)$ & 3493 (64.5\%) & \\
\hline Age & & & & & & $<0.0001$ \\
\hline $45-54$ years & $46,981(52.8 \%)$ & $4410(39.9 \%)$ & $1683(31.5 \%)$ & $1680(38.6 \%)$ & $1783(32.9 \%)$ & \\
\hline $55-64$ years & $41,945(47.2 \%)$ & $6647(60.1 \%)$ & 3665 (68.5\%) & $2668(61.4 \%)$ & $3632(67.1 \%)$ & \\
\hline Country of birth & & & & & & 0.35 \\
\hline Australia & $66,457(74.7 \%)$ & 8807 (79.7\%) & $4072(76.1 \%)$ & $3056(70.3 \%)$ & 4035 (74.5\%) & \\
\hline Others & $22,055(24.8 \%)$ & $2192(19.8 \%)$ & $1250(23.4 \%)$ & $1268(29.2 \%)$ & $1348(24.9 \%)$ & \\
\hline Missing & $414(0.5 \%)$ & $58(0.5 \%)$ & $26(0.5 \%)$ & $24(0.6 \%)$ & $32(0.6 \%)$ & \\
\hline Income & & & & & & $<0.0001$ \\
\hline$<20,000$ AUD & $5722(6.4 \%)$ & 867 (7.8\%) & $471(8.8 \%)$ & $496(11.4 \%)$ & $1096(20.2 \%)$ & \\
\hline 20,000-39,999 AUD & $11,612(13.1 \%)$ & $1574(14.2 \%)$ & $815(15.2 \%)$ & $676(15.5 \%)$ & $1138(21.0 \%)$ & \\
\hline 40,000-69,999 AUD & $20,276(22.8 \%)$ & $2554(23.1 \%)$ & $1262(23.6 \%)$ & $1004(23.1 \%)$ & $982(18.1 \%)$ & \\
\hline$\geq 70,000$ AUD & $35,585(40.0 \%)$ & $4024(36.4 \%)$ & 1920 (35.9\%) & $1343(30.9 \%)$ & $1050(19.4 \%)$ & \\
\hline Missing & $15,731(17.7 \%)$ & $2038(18.4 \%)$ & $880(16.5 \%)$ & $829(19.1 \%)$ & $1149(21.2 \%)$ & \\
\hline Education level & & & & & & $<0.0001$ \\
\hline$<10$ years & $5416(6.1 \%)$ & $729(6.6 \%)$ & $473(8.8 \%)$ & $510(11.7 \%)$ & $731(13.5 \%)$ & \\
\hline High school/TAFE & $54,865(61.7 \%)$ & $7043(63.7 \%)$ & $3373(63.1 \%)$ & $2731(62.8 \%)$ & 3605 (66.6\%) & \\
\hline University or higher & $27,812(31.3 \%)$ & $3202(29.0 \%)$ & $1461(27.3 \%)$ & $1057(24.3 \%)$ & 1003 (18.5\%) & \\
\hline Missing & $833(0.9 \%)$ & $83(0.8 \%)$ & $41(0.8 \%)$ & $50(1.1 \%)$ & $76(1.4 \%)$ & \\
\hline Body mass index ${ }^{\mathrm{b}}$ & & & & & & $<0.0001$ \\
\hline $15-180.4 \mathrm{~kg} / \mathrm{m}^{2}$ & $870(1.0 \%)$ & $114(1.0 \%)$ & $52(1.0 \%)$ & $19(0.4 \%)$ & $50(0.9 \%)$ & \\
\hline $180.5-240.9 \mathrm{~kg} / \mathrm{m}^{2}$ & $33,065(37.2 \%)$ & $4065(36.8 \%)$ & $1515(28.3 \%)$ & $743(17.1 \%)$ & $1362(25.2 \%)$ & \\
\hline $25-290.9 \mathrm{~kg} / \mathrm{m}^{2}$ & $33,145(37.3 \%)$ & $4077(36.9 \%)$ & $2248(42.0 \%)$ & $1463(33.6 \%)$ & $1852(34.2 \%)$ & \\
\hline$\geq 30 \mathrm{~kg} / \mathrm{m}^{2}$ & $16,877(19.0 \%)$ & $2169(19.6 \%)$ & $1274(23.8 \%)$ & $1854(42.6 \%)$ & $1774(32.8 \%)$ & \\
\hline Missing & 4969 (5.6\%) & $632(5.7 \%)$ & $259(4.8 \%)$ & $269(6.2 \%)$ & $377(7.0 \%)$ & \\
\hline Psychological distress $^{c}$ & & & & & & $<0.0001$ \\
\hline Low & $38,792(43.6 \%)$ & $4737(42.8 \%)$ & $2143(40.1 \%)$ & $1755(40.4 \%)$ & $2000(36.9 \%)$ & \\
\hline Mild & $32,925(37.0 \%)$ & $4098(37.1 \%)$ & 2031 (38.0\%) & $1586(36.5 \%)$ & 1967 (36.3\%) & \\
\hline Moderate & $11,051(12.4 \%)$ & $1409(12.7 \%)$ & $726(13.6 \%)$ & $552(12.7 \%)$ & $815(15.1 \%)$ & \\
\hline High & $2957(3.3 \%)$ & $368(3.3 \%)$ & $207(3.9 \%)$ & $206(4.7 \%)$ & $280(5.2 \%)$ & \\
\hline Missing & $3201(3.6 \%)$ & $445(4.0 \%)$ & $241(4.5 \%)$ & $249(5.7 \%)$ & $353(6.5 \%)$ & \\
\hline Hypertension & & & & & & $<0.0001$ \\
\hline No & $69,427(78.1 \%)$ & $8297(75.0 \%)$ & $3074(57.5 \%)$ & $2175(50.0 \%)$ & $3433(63.4 \%)$ & \\
\hline Yes & 19,499 (21.9\%) & $2760(25.0 \%)$ & $2274(42.5 \%)$ & $2173(50.0 \%)$ & $1982(36.6 \%)$ & \\
\hline Dyslipidaemia & & & & & & $<0.0001$ \\
\hline No & $81,790(92.0 \%)$ & $10,146(91.8 \%)$ & $4026(75.3 \%)$ & $3225(74.2 \%)$ & 4497 (83.0\%) & \\
\hline Yes & $7136(8.0 \%)$ & $911(8.2 \%)$ & $1322(24.7 \%)$ & $1123(25.8 \%)$ & $918(17.0 \%)$ & \\
\hline Asthma & & & & & & 0.0001 \\
\hline No & $81,319(91.4 \%)$ & $10,033(90.7 \%)$ & $4893(91.5 \%)$ & $3976(91.4 \%)$ & $4798(88.6 \%)$ & \\
\hline Yes & $7607(8.6 \%)$ & $1024(9.3 \%)$ & $455(8.5 \%)$ & $372(8.6 \%)$ & $617(11.4 \%)$ & \\
\hline Family history of depression & & & & & & 0.0674 \\
\hline No & $79,042(88.9 \%)$ & $9760(88.3 \%)$ & $4793(89.6 \%)$ & 3967 (91.2\%) & $4792(88.5 \%)$ & \\
\hline Yes & $9884(11.1 \%)$ & $1297(11.7 \%)$ & $555(10.4 \%)$ & $381(8.8 \%)$ & $623(11.5 \%)$ & \\
\hline Family history of diabetes & & & & & & $<0.0001$ \\
\hline No & $68,651(77.2 \%)$ & $8664(78.4 \%)$ & $4125(77.1 \%)$ & $2011(46.3 \%)$ & $4040(74.6 \%)$ & \\
\hline Yes & $20,275(22.8 \%)$ & $2393(21.6 \%)$ & $1223(22.9 \%)$ & $2337(53.7 \%)$ & $1374(25.4 \%)$ & \\
\hline Family history of cancer & & & & & & 0.90 \\
\hline No & $50,214(56.5 \%)$ & $5385(48.7 \%)$ & $3102(58.0 \%)$ & $2643(60.8 \%)$ & $3048(56.3 \%)$ & \\
\hline Yes & $38,712(43.5 \%)$ & $5672(51.3 \%)$ & $2246(42.0 \%)$ & $1705(39.2 \%)$ & $2366(43.7 \%)$ & \\
\hline Family history of heart disease & & & & & & $<0.0001$ \\
\hline No & $52,477(59.0 \%)$ & $6224(56.3 \%)$ & $2024(37.8 \%)$ & $2385(54.9 \%)$ & $2952(54.5 \%)$ & \\
\hline Yes & $36,449(41.0 \%)$ & $4833(43.7 \%)$ & $3324(62.2 \%)$ & $1963(45.1 \%)$ & $2461(45.4 \%)$ & \\
\hline Family history of stroke & & & & & & $<0.0001$ \\
\hline No & $68,381(76.9 \%)$ & $8335(75.4 \%)$ & $3886(72.7 \%)$ & $3248(74.7 \%)$ & $4005(74.0 \%)$ & \\
\hline Yes & $20,545(23.1 \%)$ & $2722(24.6 \%)$ & $1462(27.3 \%)$ & $1100(25.3 \%)$ & $1408(26.0 \%)$ & \\
\hline Family history of hypertension & & & & & & $<0.0001$ \\
\hline No & $41,175(46.3 \%)$ & $5031(45.5 \%)$ & $2204(41.2 \%)$ & $1831(42.1 \%)$ & $2328(43.0 \%)$ & \\
\hline Yes & $47,751(53.7 \%)$ & $6026(54.5 \%)$ & $3144(58.8 \%)$ & $2517(57.9 \%)$ & $3085(57.0 \%)$ & \\
\hline Family history of hip fracture & & & & & & $<0.0001$ \\
\hline No & $81,402(91.5 \%)$ & $10,037(90.8 \%)$ & $4879(91.2 \%)$ & $4002(92.0 \%)$ & $4818(89.0 \%)$ & \\
\hline Yes & $7524(8.5 \%)$ & $1020(9.2 \%)$ & $469(8.8 \%)$ & $346(8.0 \%)$ & $595(11.0 \%)$ & \\
\hline Family history of Parkinson's disease & & & & & & 0.95 \\
\hline No & $85,098(95.7 \%)$ & $10,584(95.7 \%)$ & $5142(96.1 \%)$ & $4173(96.0 \%)$ & $5160(95.3 \%)$ & \\
\hline Yes & $3828(4.3 \%)$ & $473(4.3 \%)$ & $206(3.9 \%)$ & $175(4.0 \%)$ & $254(4.7 \%)$ & \\
\hline Family history of dementia & & & & & & $<0.0001$ \\
\hline No & $75,675(85.1 \%)$ & $9200(83.2 \%)$ & 4540 (84.9\%) & $3725(85.7 \%)$ & 4423 (81.7\%) & \\
\hline Yes & $13,251(14.9 \%)$ & $1857(16.8 \%)$ & $808(15.1 \%)$ & $623(14.3 \%)$ & $991(18.3 \%)$ & \\
\hline
\end{tabular}

a Healthy participants were those without any of the four predefined chronic conditions at baseline.

b Body mass index was calculated as weight in kilogrammes divided by the square of height in meters.

c Psychological distress measured by the Kessler-10 scale provides a global measure of anxiety and depressive symptoms experienced in the preceding month, with the following categories: low, mild, moderate and high psychological distress. 
A

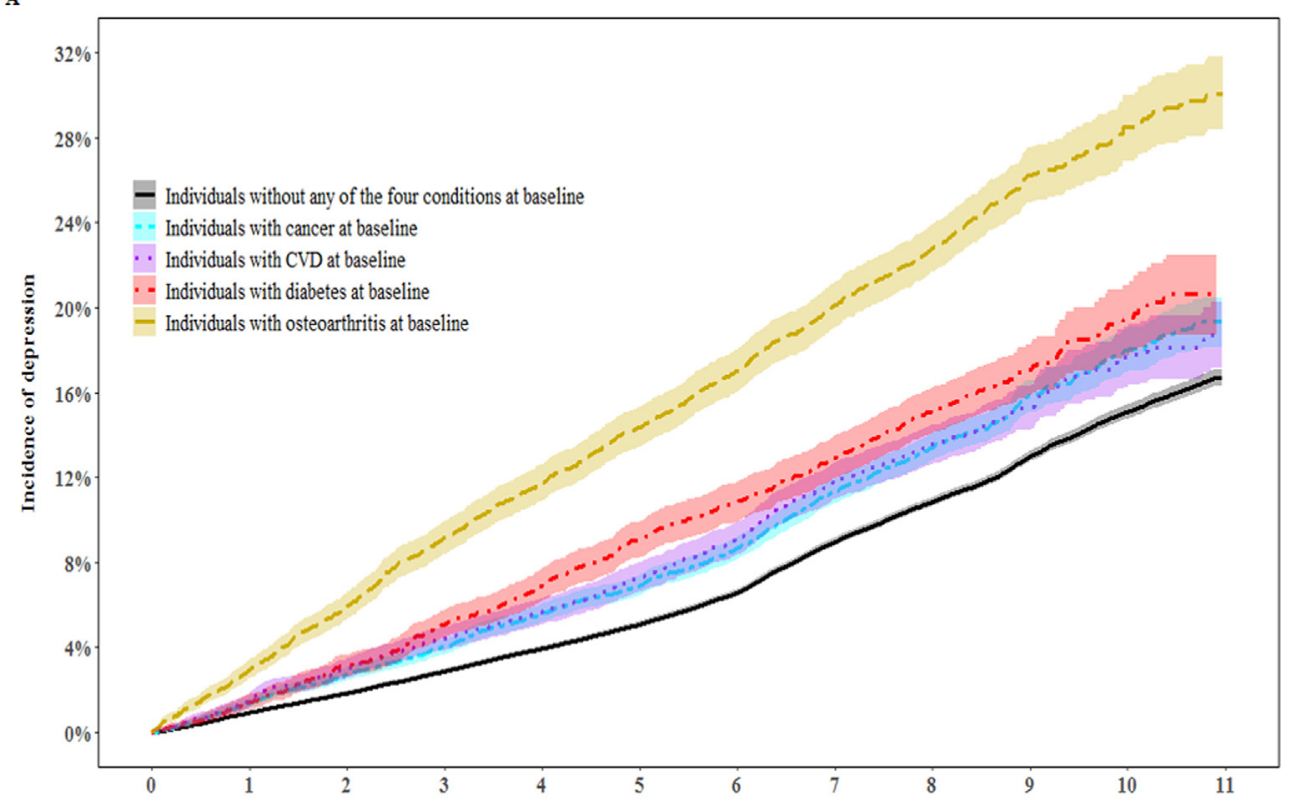

B

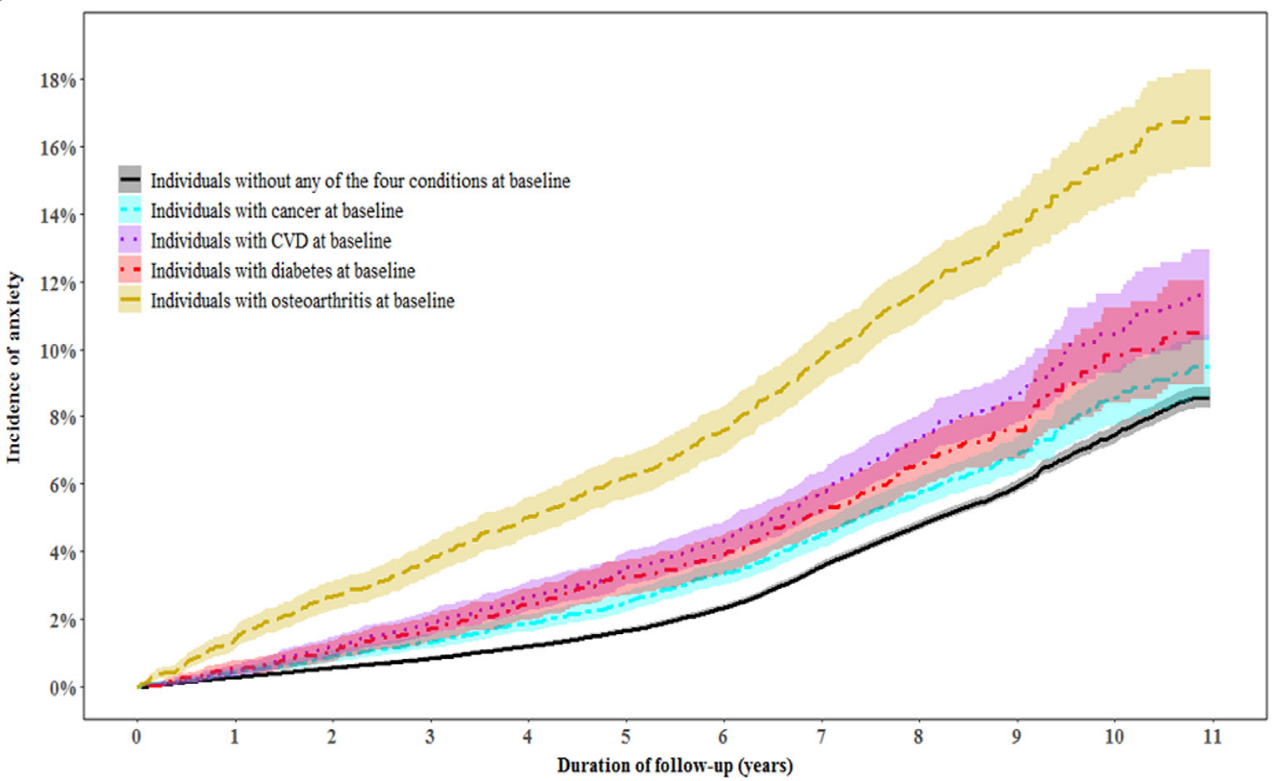

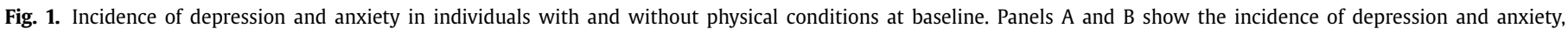

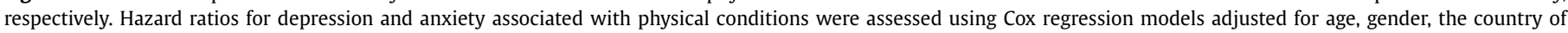

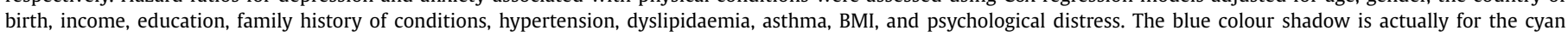

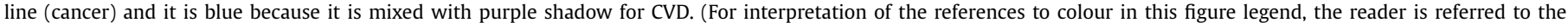
web version of this article.)

healthy population and those with cancer, CVD, diabetes, or osteoarthritis. Long-term conditions including cancer, CVD and osteoarthritis were each associated with an increased risk of incident depression and anxiety. The magnitude of the association between baseline cancer and incident depression was greater in men than in women. Diabetes was an independent risk factor for incident depression in both genders but for incident anxiety in men only. Osteoarthritis had the highest HR for both incident depression and anxiety among four conditions.

Our study agrees with previous studies showing that women had a higher incidence of depression and anxiety than men [18]. The gender gaps in incident depression and anxiety may be at- tributed to sex differences in genetics, hormones, socioeconomic status, environment, and psychological distress [23]. Our findings are independent of country of birth, age, income, education, BMI, psychological distress, chronic conditions and family history of conditions (including depression) suggesting that other potential mediators/confounders need to be investigated. One plausible explanation for the gender gaps is the lower depression recognition in men than in women [23].

Psychological consequences of cancer have been well recognised given that most people interpret a cancer diagnosis as a death warrant. We found individuals with cancer at baseline were more likely to develop depression and anxiety across different time 


\begin{tabular}{|c|c|c|c|c|c|c|c|}
\hline & \multicolumn{2}{|c|}{ Men } & \multicolumn{2}{|c|}{ Women } & \multirow{2}{*}{\multicolumn{2}{|c|}{$\begin{array}{c}\text { HR }(95 \% \mathrm{CI}) \\
\text { for women versus men* }\end{array}$}} & \multirow[t]{2}{*}{$P$ value } \\
\hline & $\begin{array}{l}\text { Events/ } \\
\text { participants }\end{array}$ & Incidence & $\begin{array}{l}\text { Events/ } \\
\text { participants }\end{array}$ & Incidence & & & \\
\hline \multicolumn{8}{|l|}{ Depression } \\
\hline Healthy population ${ }^{\dagger}$ & $3962 / 40338$ & $9 \cdot 8$ & $7176 / 48588$ & 14.8 & $\rightarrow$ & $1 \cdot 47(1 \cdot 42-1 \cdot 53)$ & $<0.0001$ \\
\hline Cancer ${ }^{\ddagger}$ & $550 / 4453$ & $12 \cdot 4$ & $1120 / 6604$ & $17 \cdot 0$ & & $1.39(1.25-1.55)$ & $<0.0001$ \\
\hline $\mathrm{CVD}^{\S}$ & $437 / 3454$ & $12 \cdot 7$ & $369 / 1894$ & $19 \cdot 5$ & & $1.55(1 \cdot 34-1 \cdot 79)$ & $<0.0001$ \\
\hline Diabetes $\|$ & $337 / 2467$ & $13 \cdot 7$ & $388 / 1881$ & $20 \cdot 6$ & & $1 \cdot 43(1 \cdot 23-1 \cdot 66)$ & $<0.0001$ \\
\hline Osteoarthritis ${ }^{\Upsilon}$ & $386 / 1922$ & $20 \cdot 1$ & $991 / 3493$ & $28 \cdot 4$ & 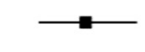 & $1 \cdot 53(1 \cdot 35-1 \cdot 72)$ & $<0.0001$ \\
\hline \multicolumn{8}{|l|}{ Anxiety } \\
\hline Healthy population & $1775 / 40338$ & $4 \cdot 4$ & $3426 / 48588$ & $7 \cdot 1$ & $\rightarrow-$ & $1 \cdot 56(1 \cdot 47-1 \cdot 66)$ & $<0.0001$ \\
\hline Cancer & $238 / 4453$ & $5 \cdot 3$ & $509 / 6604$ & $7 \cdot 7$ & $=$ & $1 \cdot 48(1 \cdot 26-1 \cdot 74)$ & $<0.0001$ \\
\hline CVD & $240 / 3454$ & $7 \cdot 0$ & $223 / 1894$ & $11 \cdot 8$ & & $-1.68(1.38-2.03)$ & $<0.0001$ \\
\hline Diabetes & $162 / 2467$ & $6 \cdot 6$ & $170 / 1881$ & $9 \cdot 0$ & & $1 \cdot 29(1 \cdot 03-1 \cdot 61)$ & 0.0024 \\
\hline Osteoarthritis & $213 / 1922$ & $11 \cdot 1$ & $513 / 3493$ & $14 \cdot 7$ & $\longrightarrow$ & $1.39(1.18-1.64)$ & $<0.0001$ \\
\hline & & & & & & $2 \cdot 0$ & \\
\hline
\end{tabular}

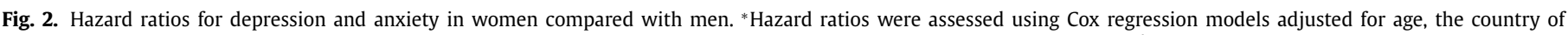

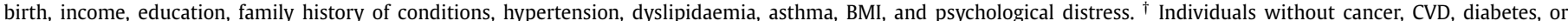

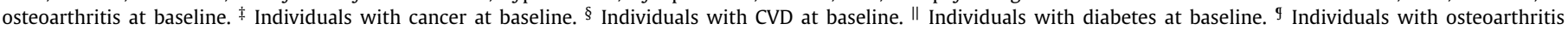
at baseline.

points during follow-up, and this association was more evident in men than in women. This is consistent with previous studies showing that cancer was a risk factor for mental disorders in both men and women $[15,24]$. Although a recent study did not analyse the interaction between gender and cancer for depression/anxiety, odds ratios (95\% CIs) for depression and anxiety associated with long-term cancer were greater in men than in in women in this study [16]. A matched study also showed that the HR for mental disorders ten years after cancer diagnosis associated with cancer was greater in men than in women [15]. However, these studies did not exclude participants with a self-reported history of mental disorders or a record of using antidepressants or anxiolytics at baseline either adjusted for important confounders including BMI, medical history, family history of chronic conditions, and psychological distress in their analysis. The composition of different types of cancer and the different associations of those cancers with incident depression/anxiety in our study may partly explain the gender difference in the magnitude of the association between cancer and depression/anxiety. Like a matched study [15], we found the HR associated with cancer decreased with increased follow-up period, which might be attributed to the fact that these cancer survivors were more likely to die during follow-up. It is also possible that the HR for depression and anxiety associated with cancer decreased with increased follow-up period if there is a favourable outcome after treatment.

We found CVD was a risk factor for incident depression and anxiety in both men and women across different time points, and this association did not differ between genders. A high prevalence of depression and anxiety has been observed in individuals with heart failure or stroke $[9,12]$; however, few studies have reported whether this prevalence was higher than that in the general population [13]. A matched study found that individuals with stroke were around two times more likely to develop depression until the second year of follow-up compared with the control group [25]. The association between depression and an increased risk of incident CVD has been well established [13], the mechanism of how CVD affects mental disorders remains to be explored. However, the perceived loss of health, functional capability and independence in CVD patients may partly explain the higher incidence of depression and anxiety in these vulnerable individuals [13]. Whether CVD was associated with an increased risk of incident anxiety has not been reported in previous studies. We found the HR for anxiety associated with CVD was greater than that for depression in both men and women suggesting anxiety also deserves concern among CVD patients.

The psychological burden imposed by awareness of having a chronic condition, its treatment, its effects on lifestyle, and shared mechanisms may partly explain the increased risks of depression in diabetes patients [26-28]. We found diabetes was an independent risk factor for incident depression in both men and women across different time points whereas diabetes was associated with an increased risk of incident anxiety in men but not women. A recent meta-analysis of 16 longitudinal studies demonstrated that diabetes was associated with an increased risk of incident depression (pooled relative risk [RR]: 1.27 (95\% CI 1.17-1.38)) [14]. Hasan et al. also reported a higher RR in studies with follow-up period $\leq$ five years than in those with follow-up period > five years, which is consistent with our findings that HR for depression associated with diabetes decreased with increased follow-up period. One prospective study showed that there was no significant association between baseline diabetes and incident anxiety, but this study is limited by a small number of diabetes patients $(n=337)$ [11]. We found the HR for incident anxiety in men decreased with an increasing follow-up period, although the association was significant throughout the follow-up. Another study also failed to find a significant association between diabetes and incident anxiety (defined by claimed data), which might be attributed to the five-year gap between baseline (1994) and follow-up (1999-2011) [29]. It is unclear why the positive association between diabetes and incident anxiety was observed in men only in our study.

We found osteoarthritis was associated with an increased risk of depression and anxiety with the highest HRs among four conditions in both men and women. A recent meta-analysis of 15,855 individuals found that around one-fifth of people were affected by depression/anxiety in those with osteoarthritis [10]. This analysis showed that osteoarthritis was not associated with an increased risk of depression or anxiety in three eligible studies [10]. A recent prospective study of 3491 participants with 4.2 years of followup demonstrated that individuals with multisite or hip/knee osteoarthritis had a higher likelihood of developing depression than those without osteoarthritis [30]. These previous studies were limited by small sample size and did not explore whether gender was 
Healthy population* With condition ${ }^{\dagger}$

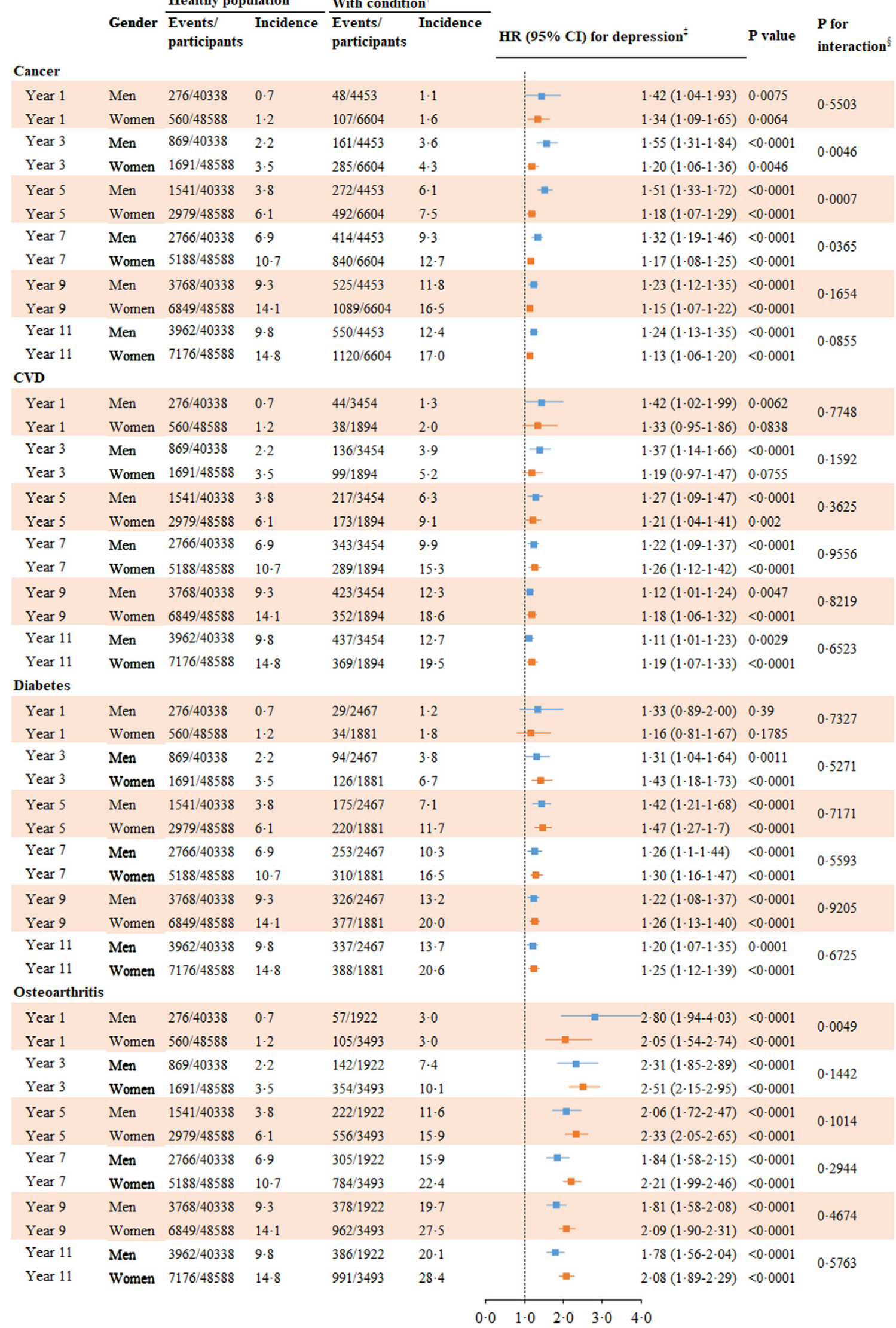

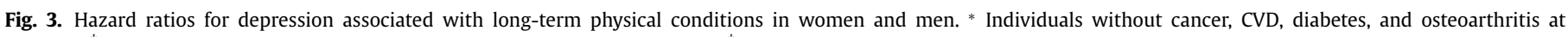

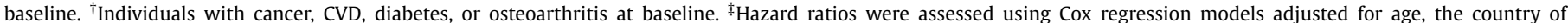

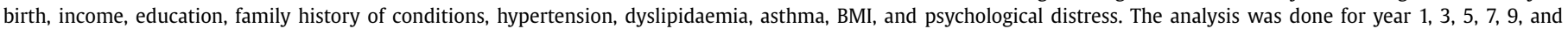
11 of follow-up. ${ }^{\S} \mathrm{P}$ value for interaction between gender and condition for incident depression. 


\begin{tabular}{|c|c|c|c|c|c|c|c|c|c|}
\hline \multirow[b]{3}{*}{ Cancer } & \multirow{3}{*}{ Gender } & \multicolumn{2}{|c|}{ Healthy population* } & \multicolumn{2}{|c|}{ With condition ${ }^{\top}$} & \multirow{2}{*}{\multicolumn{2}{|c|}{ HR $(95 \% \mathrm{CI})$ for anxiety ${ }^{\ddagger}$}} & \multirow{3}{*}{$P$ value } & \multirow{3}{*}{$\begin{array}{l}\text { P for } \\
\text { interaction }^{\S}\end{array}$} \\
\hline & & \multirow[t]{2}{*}{$\begin{array}{l}\text { Events/ } \\
\text { participants }\end{array}$} & \multirow[t]{2}{*}{ Incidence } & \multirow[t]{2}{*}{$\begin{array}{l}\text { Events/ } \\
\text { participants }\end{array}$} & \multirow[t]{2}{*}{ Incidence } & & & & \\
\hline & & & & & & & & & \\
\hline Year 1 & Men & $75 / 40338$ & $0 \cdot 2$ & $12 / 4453$ & 0.3 & 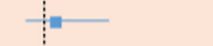 & $1.21(0.66-2.25)$ & 0.5522 & \multirow{2}{*}{0.9917} \\
\hline Year 1 & Women & $172 / 48588$ & 0.4 & $34 / 6604$ & 0.5 & $=-$ & $1.33(0.92-1.93)$ & 0.187 & \\
\hline Year 3 & Men & $227 / 40338$ & 0.6 & $48 / 4453$ & $1 \cdot 1$ & -- & $1 \cdot 71(1 \cdot 25-2 \cdot 34)$ & 0.0016 & \multirow{2}{*}{$0 \cdot 1181$} \\
\hline Year 3 & Women & $514 / 48588$ & $1 \cdot 1$ & $99 / 6604$ & 1.5 & $=$ & $1.32(1.07-1.64)$ & 0.0324 & \\
\hline Year 5 & Men & $443 / 40338$ & $1 \cdot 1$ & $80 / 4453$ & 1.8 & - & $1 \cdot 47(1-16-1 \cdot 87)$ & 0.0048 & \multirow{2}{*}{0.3203} \\
\hline Year 5 & Women & $1028 / 48588$ & $2 \cdot 1$ & $198 / 6604$ & 3.0 & $=$ & $1.33(1 \cdot 14-1 \cdot 55)$ & 0.0023 & \\
\hline Year 7 & Men & $1027 / 40338$ & $2 \cdot 6$ & $150 / 4453$ & $3 \cdot 4$ & $=$ & $1 \cdot 24(1 \cdot 05-1 \cdot 48)$ & 0.0462 & \multirow{2}{*}{0.314} \\
\hline Year 7 & Women & $2148 / 48588$ & $4 \cdot 4$ & $347 / 6604$ & $5 \cdot 3$ & $=$ & $1 \cdot 13(1 \cdot 01-1 \cdot 27)$ & 0.0493 & \\
\hline Year 9 & Men & $1638 / 40338$ & $4 \cdot 1$ & $233 / 4453$ & $5 \cdot 2$ & $=$ & $1.18(1.03-1.35)$ & 0.0496 & \multirow{2}{*}{$0 \cdot 2712$} \\
\hline Year 9 & Women & $3177 / 48588$ & 6.5 & $699 / 6604$ & $7 \cdot 6$ & 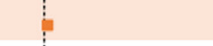 & $1.07(0.97-1.17)$ & 0.2776 & \\
\hline Year 11 & Men & $1775 / 40338$ & $4 \cdot 4$ & $238 / 4453$ & $5 \cdot 3$ & $=$ & $1 \cdot 16(1 \cdot 02-1 \cdot 33)$ & 0.0496 & \multirow{2}{*}{0.2081} \\
\hline Year 11 & Women & $3426 / 48588$ & $7 \cdot 1$ & $509 / 6604$ & $7 \cdot 7$ & 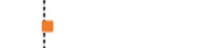 & $1.06(0.97-1.17)$ & 0.1952 & \\
\hline CVD & & & & & & & & & \\
\hline Year 1 & Men & $75 / 40338$ & $0 \cdot 2$ & $13 / 3454$ & 0.4 & $=$ & $1.31(0.70-2 \cdot 43)$ & $0-1832$ & 0.9186 \\
\hline Year 1 & Women & $172 / 48588$ & 0.4 & $13 / 1894$ & 0.7 & $=$ & $1.34(0.75-2.38)$ & $0 \cdot 188$ & 0.9180 \\
\hline Year 3 & Men & $227 / 40338$ & 0.6 & $56 / 3454$ & 1.6 & $\rightarrow-$ & $1.90(1.39-2.59)$ & $<0.0001$ & 0.2494 \\
\hline Year 3 & Women & $514 / 48588$ & $1 \cdot 1$ & $45 / 1894$ & $2 \cdot 4$ & -- & $1 \cdot 56(1 \cdot 14-2 \cdot 13)$ & 0.0014 & 0.2494 \\
\hline Year 5 & Men & $443 / 40338$ & $1 \cdot 1$ & $91 / 3454$ & $2 \cdot 6$ & - & $1.65(1.30-2 \cdot 09)$ & $<0.0001$ & 0.9251 \\
\hline Year 5 & Women & $1028 / 48588$ & $2 \cdot 1$ & $97 / 1894$ & $5 \cdot 1$ & - & $1 \cdot 71(1 \cdot 38-2 \cdot 12)$ & $<0.0001$ & 0.9231 \\
\hline Year 7 & Men & $1027 / 40338$ & $2 \cdot 6$ & $153 / 3454$ & $4 \cdot 4$ & $=$ & $1 \cdot 35(1 \cdot 13-1 \cdot 61)$ & $<0.0001$ & 0.5728 \\
\hline Year 7 & Women & $2148 / 48588$ & $4 \cdot 4$ & $154 / 1894$ & $8 \cdot 1$ & - & $1.48(1.25-1.75)$ & $<0.0001$ & $0.5 / 28$ \\
\hline Year 9 & Men & $1638 / 40338$ & $4 \cdot 1$ & $226 / 3454$ & 6.5 & $=$ & $1 \cdot 30(1-13-1-51)$ & $<0.0001$ & \\
\hline Year 9 & Women & $3177 / 48588$ & $6 \cdot 5$ & $210 / 1894$ & $11 \cdot 1$ & $=$ & $1.42(1-23-1-64)$ & $<0.0001$ & $0.51 / 2$ \\
\hline Year 11 & Men & $1775 / 40338$ & $4 \cdot 4$ & $240 / 3454$ & $7 \cdot 0$ & $=$ & $1 \cdot 30(1-13-1 \cdot 50)$ & $<0.0001$ & 0.5200 \\
\hline Year 11 & Women & $3426 / 48588$ & $7 \cdot 1$ & $223 / 1894$ & 11.8 & $=$ & $1.42(1.24-1.63)$ & $<0.0001$ & 0.5200 \\
\hline Diabetes & & & & & & & & & \\
\hline Year 1 & Men & $75 / 40338$ & $0 \cdot 2$ & $15 / 2467$ & 0.6 & $\Longrightarrow$ & $2 \cdot 10(1 \cdot 14-3 \cdot 87)$ & 0.002 & 0.0461 \\
\hline Year 1 & Women & $172 / 48588$ & 0.4 & $9 / 1881$ & 0.5 & - & $0.96(0.48-1.92)$ & 0.955 & 0.0401 \\
\hline Year 3 & Men & $227 / 40338$ & 0.6 & $37 / 2467$ & $1 \cdot 5$ & $\rightarrow-$ & $1.90(1.31-2 \cdot 77)$ & $<0.0001$ & 0.1698 \\
\hline Year 3 & Women & $514 / 48588$ & $1 \cdot 1$ & $38 / 1881$ & $2 \cdot 0$ & - & $1.22(0.86-1 \cdot 72)$ & 0.1044 & 0.1098 \\
\hline Year 5 & Men & $443 / 40338$ & $1 \cdot 1$ & $72 / 2467$ & $2 \cdot 9$ & $\rightarrow$ & $1.82(1.39-2 \cdot 38)$ & $<0.0001$ & 0.0131 \\
\hline Year 5 & Women & $1028 / 48588$ & $2 \cdot 1$ & $68 / 1881$ & $3 \cdot 6$ & 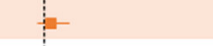 & $1.15(0.89-1.48)$ & 0.1109 & 0.0131 \\
\hline Year 7 & Men & $1027 / 40338$ & $2 \cdot 6$ & $113 / 2467$ & $4 \cdot 6$ & $=$ & $1 \cdot 38(1 \cdot 12-1 \cdot 70)$ & 0.0002 & 0.0536 \\
\hline Year 7 & Women & $2148 / 48588$ & $4 \cdot 4$ & $115 / 1881$ & $6 \cdot 1$ & - & $1.08(0.89-1.32)$ & 0.2396 & \\
\hline Year 9 & Men & $1638 / 40338$ & $4 \cdot 1$ & $154 / 2467$ & $6 \cdot 2$ & - & $1 \cdot 22(1 \cdot 02-1 \cdot 45)$ & 0.0044 & 0.0842 \\
\hline Year 9 & Women & $3177 / 48588$ & $6 \cdot 5$ & $160 / 1881$ & $8 \cdot 5$ & 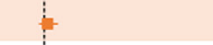 & $1.08(0.91-1.27)$ & 0.2172 & 0.0842 \\
\hline Year 11 & Men & $1775 / 40338$ & $4 \cdot 4$ & $162 / 2467$ & $6 \cdot 6$ & $=$ & $1 \cdot 21(1 \cdot 02-1 \cdot 44)$ & 0.0044 & 0.1969 \\
\hline Year 11 & Women & $3426 / 48588$ & $7 \cdot 1$ & $170 / 1881$ & $9 \cdot 0$ & 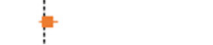 & $1.09(0.93-1.28)$ & 0.1730 & \\
\hline Osteoarth & & & & & & & & & \\
\hline Year 1 & Men & $75 / 40338$ & $0 \cdot 2$ & $25 / 1922$ & $1 \cdot 3$ & $=$ & $\rightarrow 3.08(1.77-5.38)$ & $<0.0001$ & 0.0695 \\
\hline Year 1 & Women & $172 / 48588$ & 0.4 & $52 / 3493$ & 1.5 & $\square$ & $3 \cdot 15(2 \cdot 13-4 \cdot 65)$ & $<0.0001$ & 0.0093 \\
\hline Year 3 & Men & $227 / 40338$ & 0.6 & $55 / 1922$ & $2 \cdot 9$ & $\longrightarrow$ & $2.51(1.75-3.60)$ & $<0.0001$ & 0.2233 \\
\hline Year 3 & Women & $514 / 48588$ & $1 \cdot 1$ & $151 / 3493$ & $4 \cdot 3$ & $\because-$ & $3 \cdot 22(2 \cdot 55-4 \cdot 06)$ & $<0.0001$ & 0.2233 \\
\hline Year 5 & Men & $443 / 40338$ & $1 \cdot 1$ & $88 / 1922$ & $4 \cdot 6$ & 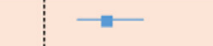 & $2 \cdot 19(1.64-2.91)$ & $<0.0001$ & 0.1267 \\
\hline Year 5 & Women & $1028 / 48588$ & $2 \cdot 1$ & $248 / 3493$ & $7 \cdot 1$ & $\rightarrow-$ & $2 \cdot 71(2-25-3-26)$ & $<0.0001$ & \\
\hline Year 7 & Men & $1027 / 40338$ & $2 \cdot 6$ & $150 / 1922$ & $7 \cdot 8$ & $\rightarrow-$ & $2 \cdot 17(1 \cdot 75-2 \cdot 70)$ & $<0.0001$ & 0.0378 \\
\hline Year 7 & Women & $2148 / 48588$ & $4 \cdot 4$ & $377 / 3493$ & $10 \cdot 8$ & $\rightarrow$ & $2 \cdot 33(2 \cdot 00-2 \cdot 71)$ & $<0.0001$ & $0.03 / 8$ \\
\hline Year 9 & Men & $1638 / 40338$ & $4 \cdot 1$ & $203 / 1922$ & $10 \cdot 6$ & - & $1.96(1.62-2-37)$ & $<0.0001$ & 0.0425 \\
\hline Year 9 & Women & $3177 / 48588$ & $6 \cdot 5$ & $489 / 3493$ & $14 \cdot 0$ & $=$ & $2.09(1.82-2.39)$ & $<0.0001$ & \\
\hline Year 11 & Men & $1775 / 40338$ & $4 \cdot 4$ & $213 / 1922$ & $11 \cdot 1$ & - & $1.95(1.62-2.35)$ & $<0.0001$ & 0.0384 \\
\hline Year 11 & Women & $3426 / 48588$ & $7 \cdot 1$ & $513 / 3493$ & $14 \cdot 7$ & $=$ & $2 \cdot 07(1 \cdot 81-2 \cdot 36)$ & $<0.0001$ & \\
\hline
\end{tabular}

Fig. 4. Hazard ratios for anxiety associated with long-term physical conditions in women and men. * Individuals without cancer, CVD, diabetes, and osteoarthritis at baseline. †Individuals with cancer, CVD, diabetes, or osteoarthritis at baseline. ${ }^{\ddagger}$ Hazard ratios were assessed using Cox regression models adjusted for age, the country of birth, income, education, family history of conditions, hypertension, dyslipidaemia, asthma, BMI, and psychological distress. The analysis was done for year 1, 3, 5, 7, 9, and 11 of follow-up. $\S \mathrm{P}$ value for interaction between gender and condition for incident depression. 
a moderator of the association between osteoarthritis and depression and anxiety. Chronic pain, disability, and decreased function caused by osteoarthritis may explain the increased risk of depression and anxiety associated with osteoarthritis [31,32]. It is also possible that osteoarthritis patients are more likely to seek help for mental disorders. There is a high prevalence of osteoarthritis in Australian adults aged $\geq 45$ years with it being more common in women (27.3\%) than in men (15.3\%) [3]. Osteoarthritis may play an important role in the development of depression and anxiety given its high prevalence [3], and its higher HR for depression and anxiety compared with other conditions as shown in our study. The gender gap in the prevalence of mental disorders in general population may be partly explained by the higher prevalence of osteoarthritis in women than in men [3].

To our knowledge, this is the first study to comprehensively explore whether long-term chronic conditions (cancer, CVD, diabetes, and osteoarthritis) were associated with increased risks of incident depression and anxiety in a community-dwelling population with a large sample size and long-term follow-up. We also uniquely examined whether the associations differed by genders and across various time points.

Several limitations need to be considered in this study. Firstly, incident depression/anxiety was identified by treatment using medications, thus possibly resulting in misclassification as well as an underestimated incidence. Furthermore, death data were not available at the moment such that the competing risk might have biased our findings. However, we examined the associations between chronic conditions at baseline and incident depression/anxiety at year $1,3,5,7,9$, and 11 of follow-up, and the results were consistent across different time points. Although the participation rate $(18 \%)$ of our study is relatively low, it is similar to other cohort studies of this kind $[33,34]$. It has also been reported that similar associations between exposures and health outcomes in this cohort study compared with a population representative study [20].

In conclusion, women had a higher incidence of depression and anxiety than men and this association was independent of chronic conditions. Osteoarthritis, CVD, and cancer were independent risk factors for depression and anxiety in both genders. Individuals with long-term osteoarthritis were at most risk of depression and anxiety compared with healthy population and those with other conditions.

\section{Contributors}

XS, LZ, and MH conceived and designed the research. XS and LZ conducted data analysis and interpretation. XS wrote the initial draft of the manuscript. XS, LZ, WP, EH, CS, and MH revised the manuscript. All authors read and approved the final manuscript.

\section{Funding}

Professor Mingguang He receives support from the University of Melbourne at Research Accelerator Program and the CERA Foundation. The Centre for Eye Research Australia (CERA) receives Operational Infrastructure Support from the Victorian State Government. The specific project is funded by Australia China Research Accelerator Program at CERA. Professor Mingguang He is also supported by the Fundamental Research Funds of the State Key Laboratory in Ophthalmology, National Natural Science Foundation of China (81420108008). The sponsor or funding organisation had no role in the design or conduct of this research.

\section{Declaration of Competing Interest}

We declare no competing interests.

\section{Acknowledgements}

This research was completed using data collected through the 45 and Up Study (www.saxinstitute.org.au). The 45 and Up Study is managed by the Sax Institute in collaboration with major partner Cancer Council NSW; and partners: the National Heart Foundation of Australia (NSW Division); NSW Ministry of Health; NSW Government Family \& Community Services-Ageing, Carers and the Disability Council NSW; and the Australian Red Cross Blood Service. We thank the other investigators, staff, and participants of the 45 and Up Study cohort for their important contributions.

\section{Appendix A. Supplementary Data}

Supplementary data to this article can be found online at https://doi.org/10.1016/j.eclinm.2019.08.010.

\section{References}

[1] Wang H, Naghavi M, Allen C, et al. Global, regional, and national life expectancy, all-cause mortality, and cause-specific mortality for 249 causes of death, 1980-2015: a systematic analysis for the global burden of disease study 2015. Lancet 2016:388:1459-544.

[2] Australian Institute of Health and Welfare. Australia's health 2018-in brief. Canberra: Australian Institute of Health and Welfare; 2018.

[3] Australian Bureau of Statistics. National health survey: first results, 2014-15. Canberra: Australian Bureau of Statistics; 2015.

[4] Vos T, Flaxman AD, Naghavi M, et al. Years lived with disability (YLDs) for 1160 sequelae of 289 diseases and injuries 1990-2010: a systematic analysis for the global burden of disease study 2010. Lancet 2012;380:2163-96.

[5] Kisely S, Crowe E, Lawrence D. Cancer-related mortality in people with mental illness. JAMA Psychiat 2013;70:209-17.

[6] Correll CU, Solmi M, Veronese N, et al. Prevalence, incidence and mortality from cardiovascular disease in patients with pooled and specific severe mental illness: a large-scale meta-analysis of 3,211,768 patients and 113,383,368 controls. World psychiatry : official journal of the World Psychiatric Association (WPA) 2017;16:163-80

[7] van Dooren FE, Nefs G, Schram MT, Verhey FR, Denollet J, Pouwer F. Depression and risk of mortality in people with diabetes mellitus: a systematic review and meta-analysis. PLoS One 2013;8:e57058.

[8] Scott KM, Von Korff M, Alonso J, et al. Mental-physical co-morbidity and its relationship with disability: results from the world mental health surveys. Psychol Med 2009;39:33-43.

[9] Mitchell AJ, Sheth B, Gill J, et al. Prevalence and predictors of post-stroke mood disorders: a meta-analysis and meta-regression of depression, anxiety and adjustment disorder. Gen Hosp Psychiatry 2017;47:48-60.

[10] Stubbs B, Aluko Y, Myint PK, Smith TO. Prevalence of depressive symptoms and anxiety in osteoarthritis: a systematic review and meta-analysis. Age Ageing 2016;45:228-35.

[11] Engum A. The role of depression and anxiety in onset of diabetes in a large population-based study. J Psychosom Res 2007;62:31-8.

[12] Yohannes AM, Willgoss TG, Baldwin RC, Connolly MJ. Depression and anxiety in chronic heart failure and chronic obstructive pulmonary disease: prevalence, relevance, clinical implications and management principles. Int J Geriatr Psychiatry 2010:25:1209-21.

[13] Hare DL, Toukhsati SR, Johansson P, Jaarsma T. Depression and cardiovascular disease: a clinical review. Eur Heart J 2014;35:1365-72.

[14] Hasan SS, Mamun AA, Clavarino AM, Kairuz T. Incidence and risk of depression associated with diabetes in adults: evidence from longitudinal studies. Community Ment Health J 2015;51:204-10.

[15] Lu D, Andersson TM, Fall K, et al. Clinical diagnosis of mental disorders immediately before and after cancer diagnosis: a nationwide matched cohort study in Sweden. JAMA Oncol 2016;2:1188-96.

[16] Hawkins NA, Soman A, Buchanan Lunsford N, Leadbetter S, Rodriguez JL. Use of medications for treating anxiety and depression in cancer survivors in the United States. J Clin Oncol 2017:35:78-85.

[17] Clarke DM, Currie KC. Depression, anxiety and their relationship with chronic diseases: a review of the epidemiology, risk and treatment evidence. Med J Aust 2009:190:S54-60.

[18] Steel Z, Marnane C, Iranpour C, et al. The global prevalence of common mental disorders: a systematic review and meta-analysis 1980-2013. Int J Epidemiol 2014;43:476-93.

[19] Banks E, Redman S, Jorm L, et al. Cohort profile: the 45 and up study. Int J Epidemiol 2008;37:941-7

[20] Mealing NM, Banks E, Jorm LR, Steel DG, Clements MS, Rogers KD. Investigation of relative risk estimates from studies of the same population with contrasting response rates and designs. BMC Med Res Methodol 2010;10:26.

[21] Kessler RC, Andrews G, Colpe LJ, et al. Short screening scales to monitor population prevalences and trends in non-specific psychological distress. Psychol Med 2002;32:959-76. 
[22] Benjamini Y. Y H. controlling the false discovery rate: a practical and powerful approach to multiple testing. J R Stat Soc Ser 1995;57:289-300.

[23] Kuehner C. Why is depression more common among women than among men? Lancet Psychiatry 2017;4:146-58.

[24] Pitman A, Suleman S, Hyde N, Hodgkiss A. Depression and anxiety in patients with cancer. BMJ 2018;361:k1415.

[25] Jorgensen TS, Wium-Andersen IK, Wium-Andersen MK, et al. Incidence of depression after stroke, and associated risk factors and mortality outcomes, in a large cohort of Danish patients. JAMA Psychiat 2016;73:1032-40.

[26] Snoek FJ, Bremmer MA, Hermanns N. Constructs of depression and distress in diabetes: time for an appraisal. The lancet Diabetes \& endocrinology 2015;3:450-60.

[27] Katon WJ, Russo JE, Heckbert SR, et al. The relationship between changes in depression symptoms and changes in health risk behaviors in patients with diabetes. Int J Geriatr Psychiatry 2010;25:466-75.

[28] Moulton CD, Pickup JC, Ismail K. The link between depression and diabetes: the search for shared mechanisms. The lancet Diabetes \& endocrinology 2015;3:461-71.

[29] Marrie RA, Patten SB, Greenfield J, et al. Physical comorbidities increase the risk of psychiatric comorbidity in multiple sclerosis. Brain and behavior 2016; e00493:6.
[30] Veronese N, Stubbs B, Solmi M, et al. Association between lower limb osteoarthritis and incidence of depressive symptoms: data from the osteoarthritis initiative. Age Ageing 2017;46:470-6.

[31] Wilkie R, Blagojevic-Bucknall M, Jordan KP, Lacey R, McBeth J. Reasons why multimorbidity increases the risk of participation restriction in older adults with lower extremity osteoarthritis: a prospective cohort study in primary care. Arthritis Care Res (Hoboken) 2013;65:910-19.

[32] Messier SP, Mihalko SL, Legault C, et al. Effects of intensive diet and exercise on knee joint loads, inflammation, and clinical outcomes among overweight and obese adults with knee osteoarthritis: the IDEA randomized clinical trial. JAMA 2013;310:1263-73.

[33] Farrell L, Hollingsworth B, Propper C, Shields MA. The socioeconomic gradient in physical inactivity: evidence from one million adults in England. Soc Sci Med 2014;123:55-63.

[34] Nohr EA, Frydenberg M, Henriksen TB, Olsen J. Does low participation in cohort studies induce bias? Epidemiology 2006;17:413-18. 


\section{University Library}

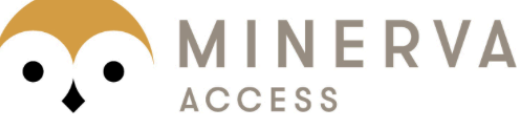

A gateway to Melbourne's research publications

Minerva Access is the Institutional Repository of The University of Melbourne

Author/s:

Shang, X;Peng, W;Hill, E;Szoeke, C;He, M;Zhang, L

Title:

Incidence of Medication-Treated Depression and Anxiety Associated with Long-Term Cancer, Cardiovascular Disease, Diabetes and Osteoarthritis in Community-dwelling Women and Men

Date:

2019-10-01

Citation:

Shang, X., Peng, W., Hill, E., Szoeke, C., He, M. \& Zhang, L. (2019). Incidence of Medication-Treated Depression and Anxiety Associated with Long-Term Cancer, Cardiovascular Disease, Diabetes and Osteoarthritis in Community-dwelling Women and Men. ECLINICALMEDICINE, 15, pp.23-32. https://doi.org/10.1016/j.eclinm.2019.08.010.

Persistent Link:

http://hdl.handle.net/11343/246690

License:

CC BY-NC-ND 